\title{
The RoPE Score and Right-to-Left Shunt Severity by Transcranial Doppler in the CODICIA Study
}

\author{
Benjamin S. Wessler ${ }^{a, b}$ David M. Kent ${ }^{b, c}$ David E. Thaler ${ }^{c}$ Robin Ruthazer ${ }^{b}$ \\ Jennifer S. Lutz ${ }^{\text {b Joaquín Serena }}{ }^{d}$ \\ a Division of Cardiology, Tufts Medical Center, ${ }^{b}$ Predictive Analytics and Comparative Effectiveness Center, Institute \\ for Clinical Research and Health Policy Studies and 'Department of Neurology, Tufts Medical Center/Tufts University \\ School of Medicine, Boston, Mass., USA; ${ }^{d}$ Department of Neurology, Hospital Universitari Doctor Josep Trueta \\ Institut d'Investigació Biomèdica de Girona, Girona, Spain
}

\section{Key Words}

Cerebrovascular disease - Stroke - Doppler - Cryptogenic stroke $\cdot$ Patent foramen ovale $\cdot$ Echocardiography

\begin{abstract}
Background: For patients with cryptogenic stroke (CS) and patent foramen ovale (PFO), it is unknown whether the magnitude of right-to-left shunt (RLSh) measured by contrast transcranial Doppler (c-TCD) is correlated with the likelihood an identified PFO is related to CS as determined by the Risk of Paradoxical Embolism (RoPE) score. Additionally, for patients with CS, it is unknown whether PFO assessment by c-TCD is more sensitive for identifying RLSh compared with transesophageal echocardiography (TEE). Our aim was to determine the significance of RLSh grade by C-TCD in patients with PFO and CS. Methods: We evaluated patients with CS who had RLSh quantified by C-TCD in the Multicenter Study into RLSh in Cryptogenic Stroke (CODICIA) to determine whether there is an association between C-TCD shunt grade and the RoPE Score. For patients who underwent C-TCD and TEE, we determined whether there is agreement in identifying and grading RLSh between these two modalities. Results: The RoPE score predicted the presence versus the absence of RLSh documented by c-TCD (c-statistic =
\end{abstract}

0.66). For patients with documented RLSh by c-TCD, shunt severity was correlated with increasing RoPE score (rank correlation $(r)=0.15, p=0.01)$. Among 293 patients who had both c-TCD and TEE performed, c-TCD was more sensitive (98.7\%) for detecting RLSh. Of the 97 patients with no PFO identified on TEE, 28 (29\%) had a large amount of RLSh seen on C-TCD. Conclusions: For patients with CS, severity of RLSh by c-TCD is positively correlated with the RoPE score, indicating that this technique for shunt grading identifies patients more likely to have pathogenic rather than incidental PFOs. c-TCD is also more sensitive in detecting RLSh than TEE. These findings suggest an important role for C-TCD in the evaluation of PFO in the setting of CS. $02015 \mathrm{~s}$. Karger AG, Basel

\section{Introduction}

Patent foramen ovale (PFO) is seen in approximately $25 \%$ of adults and generally is considered a normal anatomic variant [1]. Nonetheless, a consistent association between PFO and cryptogenic stroke (CS) has been observed [2]. However, given that PFO is common in the general population and that CS can be caused by several different occult stroke mechanisms, a PFO discovered in

\section{KARGER 125}

(c) 2015 S. Karger AG, Base

1015-9770/15/0402-0052\$39.50/0

E-Mail karger@karger.com

www.karger.com/ced
David M. Kent, MD, MS

Predictive Analytics and Comparative Effectiveness Center

Institute of Clinical Research and Health Policy Studies, Tufts Medical Center 800 Washington Street, Box 63, Boston, MA 02111 (USA)

E-Mail dkent1@tuftsmedicalcenter.org 
Table 1. RoPE score calculator. Clinical characteristics used to estimate the likelihood of an observed PFO is related to CS (adopted from [9])

\begin{tabular}{lll}
\hline Characteristic & Points & RoPE score \\
\hline No history of hypertension & 1 \\
No history of diabetes & 1 \\
No history of stroke or TIA & 1 \\
Non-smoker & 1 \\
Cortical infarct on imaging & 1 \\
Age, years & \\
$18-29$ & 5 \\
$30-39$ & 4 \\
$40-49$ & 3 \\
$50-59$ & 2 \\
$60-69$ & 1 \\
$\geq 70$ & 0
\end{tabular}

Total score (sum of individual points)

Maximum score $\mathrm{a}^{\mathrm{a}}$

Minimum score ${ }^{\mathrm{b}}$
10 a Patient $>30$ years with no hypertension, no diabetes, no history of stroke or TIA, non-smoker, and cortical infarct. ${ }^{b}$ Patient $\geq 70$ years with hypertension, diabetes, prior stroke, current smoker, and no cortical infarct.

a patient with CS may be an incidental finding unrelated to the index stroke, and not linked to the risk of recurrence [3]. Heterogeneity of stroke mechanisms among patients with PFO and CS likely explains, in part, why three recent randomized controlled trials of patients with $\mathrm{CS}$ and PFO showed no difference in their primary intention to treat outcomes for patients randomized to either device-based closure or medical therapy [4-7].

It has recently become clear that for patients with CS and $\mathrm{PFO}$, the probability that an identified PFO is an incidental finding is related to patient-specific characteristics [8]. We reported the Risk of Paradoxical Embolism (RoPE) score (table 1), a tool that stratifies patients with $\mathrm{CS}$ according to the probability of finding a PFO. The score is based on the strong and consistent empirical relationship between easily obtainable clinical variables and the prevalence of a PFO in CS patients. Bayes' theorem relates the score to the PFO-attributable fraction - that is, an estimate of the probability that an observed PFO is related to an index CS rather than being an incidental finding [9]. Briefly, for patients with CS and PFO, younger patients without risk factors for atherosclerotic disease (i.e. hypertension, diabetes, smoking) or a prior stroke and with a visible superficial lesion on neuroimaging are more likely to have a PFO-attributable CS (high RoPE score). Conversely, for older patients with more tradi- tional risk factors for ischemic stroke and without a cortical infarct on imaging, an observed PFO is more likely to be an incidental finding (low RoPE score).

While the RoPE score stratifies patients with CS based on clinical variables, it is unknown whether anatomic or physiologic features of the PFO itself are associated with the likelihood that an observed PFO is pathogenically related to CS. Several anatomic features have been proposed as identifying higher risk PFOs, including the presence of an atrial septal aneurysm [10], large shunt size [11], and the presence of a right-to-left shunt at rest [12]. Prior studies, however, have found poor interobserver agreement in identifying these features with transesophageal echocardiography (TEE) [13], long considered the gold standard for investigating PFOs [14]. Further, we recently reported that these purported 'high risk' TEE features, as measured in the component RoPE databases, do not correlate with the likelihood that an observed PFO is related to CS (as estimated by the RoPE score) [15]. These observations suggest that better tools are needed to evaluate the risk associated with PFOs.

Contrast transcranial Doppler (c-TCD) is an alternative technique for quantifying right-to-left shunt (RLSh) $[16,17]$. This technique has fair concordance with TEE for detection of RLSh, and a recent meta-analysis has reported a c-TCD sensitivity of $97 \%$ and specificity of $93 \%$ for detection of RLSh $[18,19]$. Although the ability and relevance of RLSh grade evaluated by c-TCD are unknown, some authors have recommended that c-TCD be the first-line screening test to detect RLSh for patients with CS [20]. In this study, we investigate whether shunt grade determined by c-TCD is correlated with RoPE score, and also whether c-TCD and TEE agree with respect to shunt identification and grading.

\section{Methods}

We analyzed patient level data from the Multicenter Study into RLSh in Cryptogenic Stroke (CODICIA) [21]. This was a prospective, multicenter, observational study that took place from 2000 to 2005 in 17 Spanish neurology departments. Patients were at least 18 years old and had a recent diagnosis of CS. c-TCD was the prespecified screening test for RLSh detection.

The c-TCD protocol used in the CODICIA database has been previously reported [21]. Briefly, the presence or absence of RLSh was investigated with a $2-\mathrm{MHz}$ probe monitoring the middle cerebral artery (MCA) through the temporal window, consistent with current practice standards $[22,23]$. Agitated microbubble saline solution was injected in the antecubital vein through a 20-gauge/32$\mathrm{mm}$ catheter. Microbubble injection was performed 3 times at rest and 3 times during the Valsalva maneuver. The Valsalva maneuver was standardized by asking patients to blow into a manometer un- 


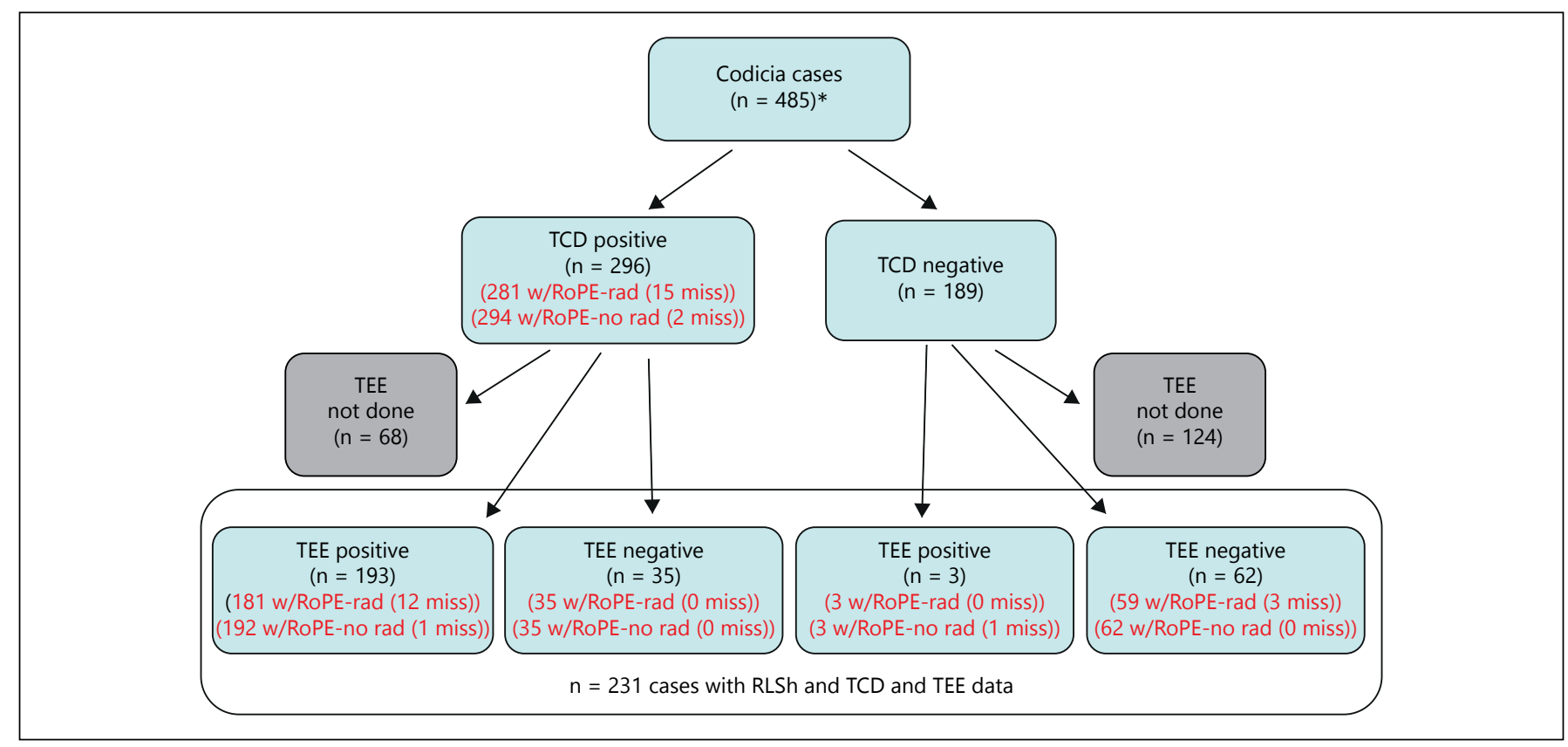

Fig. 1. Patients in the CODICIA database included in this analysis. Our primary analysis evaluated patients who had RLSh documented by c-TCD. Our secondary analyses focused on the patients with

til 50-60 $\mathrm{mm} \mathrm{Hg}$ of pressure was reached and held for at least 5-7 s. Efficacy of the Valsalva maneuver was identified by a reduction of the mean velocity in the MCA by at least $25 \%$. The magnitude of RLSh was determined by counting the number of microbubbles in the MCA in the first $7 \mathrm{~s}$ after bolus infusion. Patients were divided into 5 groups: 'normal' $c$-TCD study $=$ no microbubbles, 'small' $<10$ microbubbles, 'moderate' $=10-25$ microbubbles, 'shower' pattern $>25$ microbubbles, and 'curtain' pattern $=$ uncountable microbubbles. As originally presented, subjects in the CODICIA database were placed into 1 of 3 groups: 'no RLSh', 'nonmassive RLSh' ( $\leq 25$ microbubbles), and 'massive RLSh' (m-RLSh; corresponding to the shower and curtain patterns).

Our secondary analysis focused on participants in CODICIA who underwent both c-TCD and TEE examination either as part of the study protocol or at the discretion of the treating clinicians. Per protocol, TEE was performed when m-RLSh was detected by c-TCD [21]. As with the c-TCD exam, saline microbubbles were injected through the antecubital vein. RLSh was defined as the appearance of microbubbles in the left atrium within 3 cardiac cycles of arrival in the right atrium. The TEE protocol used in CODICIA has been previously described and is consistent with current practice standards [21,24]. TEE derived RLSh severity was determined by the maximum microbubble count in a given frame: 'no RLSh', 'small RLSh' (1-10 bubbles), 'moderate' (11-25 bubbles), and 'large' ( $>25$ bubbles). In this analysis, we examined the sensitivity of both techniques for detecting RLSh.

\section{RoPE Score}

The RoPE score predicts the likelihood of finding a PFO for patients with CS [9]. Through application of Bayes' theorem the RoPE score allows estimation of PFO attributable fraction from
RLSh who were evaluated by both c-TCD and TEE. * Original report contains 486 patients. One patient's data was closed before the incident event and as a result not included [21].

strata-specific $\mathrm{PFO}$ prevalence and the control rate PFO prevalence [8]. By using factors strongly and consistently associated with finding a PFO for an individual with CS across 12 different database, the RoPE Score permits a patient-specific attributable fraction to be empirically estimated by applying a patient-specific PFO prevalence rate. Namely, PFO prevalence in CS patients is increased in younger patients, without vascular risk factors (diabetes, hypertension, smoking) or a prior stroke or transient ischemic attack, and with a superficial lesion on neuroimaging. As previously reported, the 10-point RoPE score is calculated from a simple count of these factors [9].

\section{Analysis}

To explore whether the severity of RLSh determined by c-TCD is correlated with the RoPE score, we first evaluated the RoPE score to confirm that it effectively discriminates CODICIA patients based on the probability that they have a PFO. It should be noted that for this population, RLSh on c-TCD was used as a surrogate for the presence of PFO, originally described in the RoPE database as any right-to-left shunting seen either on c-TCD or TEE [25]. As previously reported, the RoPE score performed well in the original database and was able to predict the presence or absence of PFO in the RoPE database (c-statistic $=0.68)$ with the PFO-attributable fraction ranging from $0 \%$ (truncated lower limit for RoPE score $0-3)$ to $88 \%$ for high RoPE scores (9-10), assuming a $25 \%$ control PFO prevalence rate [9].

Following this, our primary analysis evaluated patients with RLSh in the CODICIA database investigated with c-TCD. Since the primary screening test was c-TCD, there were patients who had RLSh quantification with this technique who did not undergo TEE (fig. 1). 
Table 2. Comparison of the RoPE score, the observed probability of identifying RLSh in CODICIA population, and the predicted PFO prevalence according to RoPE score across the entire CODICIA database studied by $\mathrm{c}-\mathrm{TCD}$

\begin{tabular}{lrrr}
\hline Patients studied with c-TCD & \multicolumn{2}{c}{ RoPE score (3 groups) } \\
\cline { 2 - 4 } & $1-4$ & $5-6$ & $7-10$ \\
\hline n for analysis & 151 & 169 & 146 \\
Observed RLSh prevalence, \% & 50 & 56 & 79 \\
Predicted PFO prevalence, \% & 30 & 46 & 63 \\
\hline
\end{tabular}

Table 3. Primary analysis. Comparison of characteristics of subjects who had shunt $(\mathrm{n}=296)$ vs. no shunt $(\mathrm{n}=192)$ based on TCD result

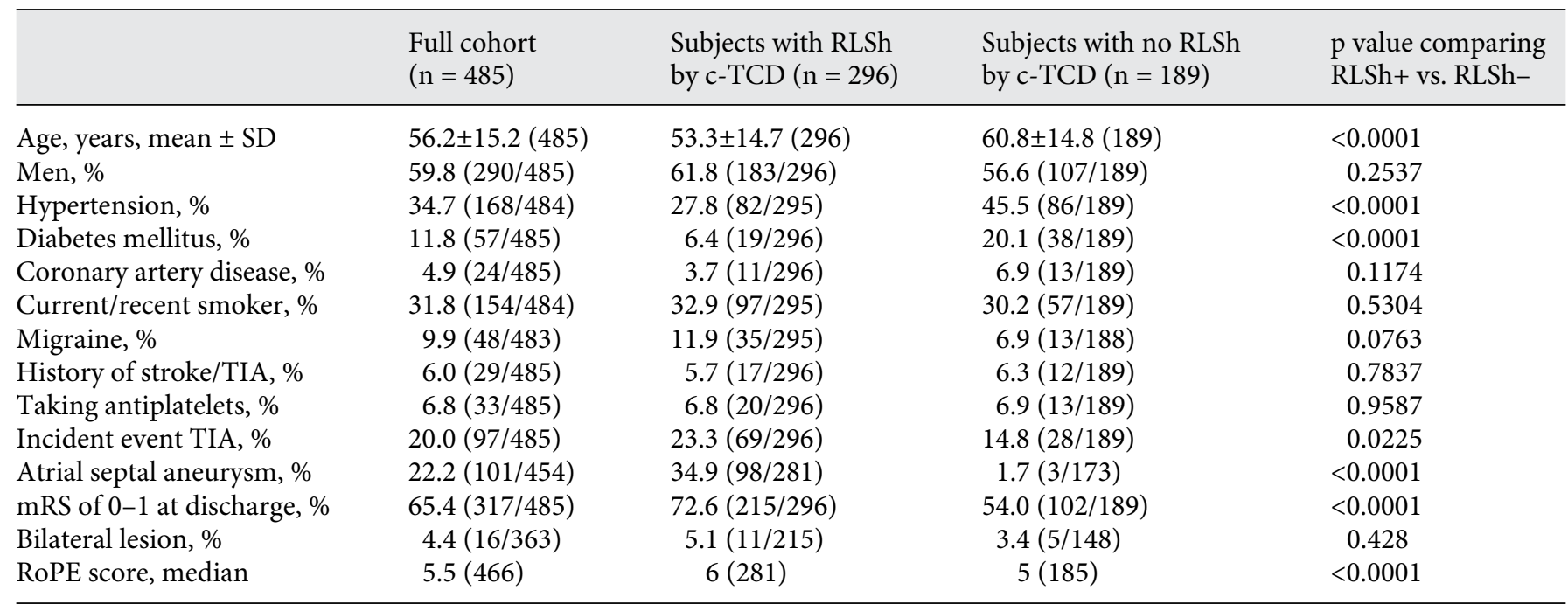

$\mathrm{mRS}=$ Modified Rankin score; TIA $=$ transient ischemic attack

We assessed whether the severity of RLSh by c-TCD correlates with the RoPE score. Our secondary analyses focused on the patients who underwent both c-TCD and TEE. We evaluated whether there is agreement between c-TCD and TEE for detection of PFO as a categorical variable (shunt vs. no shunt). We also evaluated agreement between these techniques for shunt grade represented as an ordinal variable (no shunt vs. small shunt vs. large shunt) by mapping the grading categories from each imaging technique empirically (online suppl. tables e- 3 and e-4; for all online suppl. material, see www.karger.com/ doi/10.1159/000430998). For both TEE and c-TCD, small shunts were defined as those with 10 microbubbles or fewer. For TEE evaluation, Valsalva was attempted during each examination though the presence or absence of effective Valsalva maneuver during the exam was not reported in the original dataset. For cTCD exams we evaluated the presence or absence of RLSh during Valsalva maneuver, thus representing the period of presumed maximum shunting. We determined the sensitivity of both techniques of RLSh detection by using identification of RLSh by either technique' as the gold standard method of identifying shunts and assumed $100 \%$ specificity. For the analysis of RLSh severity and RoPE score category, we calculated Spearman's rank coefficients. We created scatter plots with smoothed lines to demonstrate this association.

Significance of Right-to-Left Shunting by TCD: Analysis of the CODICIA Database

\section{Results}

The RoPE score effectively stratified the CODICIA patients according to the likelihood of finding RLSh (table 2 and online suppl. table e-1). The RoPE score c-statistic for this database was 0.66 and compares favorably to that in the overall RoPE development database (c-statistic 0.68). The PFO prevalence ranged from $50 \%$ for patients with low RoPE scores (1-4) to 79\% for high RoPE scores (710). Of the 485 patients in the CODICIA study, 296 patients were positive for RLSh by c-TCD (fig. 1). The characteristics of subjects with RLSh included in our primary analysis are shown in table 3 . When compared to patients who did not have RLSh documented by c-TCD, the patients evaluated here were younger and less likely to have the traditional stroke risk factors of hypertension or diabetes. For patients with RLSh identified with c-TCD, shunt grade determined by c-TCD was correlated with the RoPE score (rank correlation $(r)=0.15$ (95\% confidence interval (CI): 0.038, 0.268), $\mathrm{p}=0.01$ ) (fig. 2). 


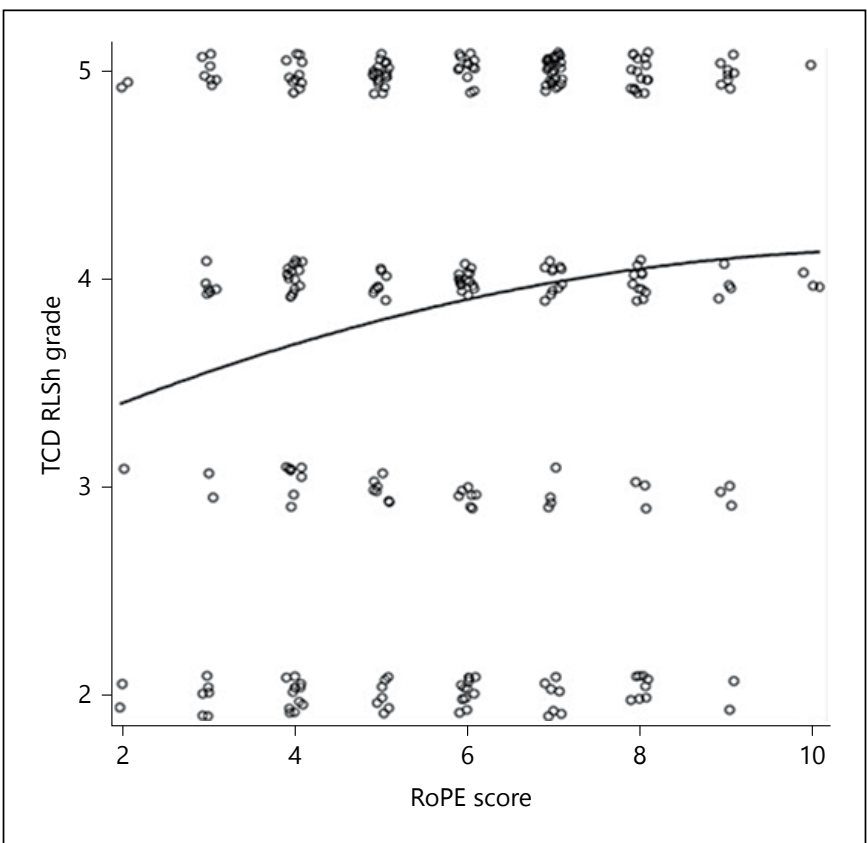

Fig. 2. Correlation between the RoPE score and shunt grade determined by c-TCD. Scatter plot with fitted penalized B-spline curve with 4 evenly spaced knots. Higher RoPE score indicates higher probability that an observed PFO is pathogenically related to CS. For c-TCD grade, microbubble count was made in the first $7 \mathrm{~s}$ after bolus ( $1=$ none, $2=1-10$ microbubbles, $3=11-25$ microbubbles, $4>25$ microbubbles, $5=$ uncountable).

Table 4. Agreement of right-to-left shunt severity determined by c-TCD and TEE

\begin{tabular}{lllll}
\hline \multirow{2}{*}{$\begin{array}{l}\text { c-TCD/ } \\
\text { Valsalva shunt }\end{array}$} & \multicolumn{2}{l}{ TEE shunt (worse from Valsalva or rest) } & \multirow{2}{*}{ Total } \\
\cline { 2 - 4 } & none (1) & small (2) & large $(3,4,5)$ & \\
\hline None (1) & 62 & 3 & 0 & 65 \\
Small (2) & 7 & 16 & 9 & 32 \\
Large (3, 4, 5) & 28 & 33 & 135 & 196 \\
\hline Total & 97 & 52 & 144 & 293 \\
\hline
\end{tabular}

Shunt size is coded: ( 1 ) is no shunt, (2) is small shunt, $(3,4$, or 5 ) is larger shunt size. Shunt severity is coded as worst shunt either during Valsalva or rest.

Our secondary analyses were limited to patients with c-TCD and TEE data (online suppl. table e-2). Of the 485 CODICIA patients, 228 had protocol-driven TEE and 65 patients who had TEE despite PFO-negative c-TCD. TEEs were not obtained for 68 patients despite RLSh positive cTCD (fig. 1). Characteristics of patients $(n=293)$ included in this secondary analysis are shown in the online supplementary table e-5. We attempted to compare these two imaging modalities directly. RLSh was seen by c-TCD in $228(78 \%)$ and TEE in $196(67 \%)$ subjects, $\kappa=0.68(95 \%$ CI: $0.59,0.77$ ) (online suppl. table e-6). There were 97 patients with no PFO identified by TEE. Of these patients, 35 (36\%) had an RLSh detected by c-TCD. Twenty-eight of these shunts $(80 \%)$ seen only with c-TCD were large in size. The sensitivities for identifying RLSh by c-TCD and TEE were 98.7 and $84.8 \%$, respectively. Overall, a large shunt was identified in 144 (49\%) of the cases by TEE and in $196(67 \%)$ of the cases by c-TCD. There was moderate agreement between shunt grades identified by these two techniques, $\kappa=0.59$ (95\% CI: 0.51, 0.67) (table 4).

\section{Discussion}

Clinical decisions for patients with CS and PFO depend on whether or not an observed PFO is believed to be pathogenically related to an index CS event. We have shown previously that patient-specific factors can help us understand this relationship [9]. However, we have not found proposed 'high-risk' features determined by TEE to be associated with the RoPE score [15]. Conversely, in this analysis of patients in the CODICIA database, we demonstrated that RoPE score predicts the likelihood of finding RLSh by c-TCD and that shunt severity determined by c-TCD is correlated with the RoPE score. Our analysis of patients who had both tests done showed that there is moderate agreement between c-TCD and TEE for identifying and grading RLSh and that there are many examples where c-TCD identified RLSh where none was seen during TEE evaluation. Moreover, a significant number of the RLSh cases identified only by c-TCD showed large shunting. Our findings suggest that c-TCD evaluation and grading of RLSh are important tools for patients with CS and complement TEE characterization of PFO.

As previously reviewed, the evaluation of shunt grade by TEE in routine practice is prone to measurement error and other limitations [15]. RLSh evaluation by c-TCD overcomes some of the limitations that accompany TEE. Notably, c-TCD is noninvasive and thus better tolerated in patients with a history of stroke or swallowing difficulties. Additionally, no sedation is needed making Valsalva maneuver more effective. Further, when patients are evaluated both with and without a TEE probe in place, the presence of a TEE probe itself may reduce the probability of achieving a right $>$ left pressure gradient during Valsalva [26]. c-TCD also permits direct detection of the 
microbubbles that flow into the cerebral circulation and as compared with TEE, there is a high level of agreement in identifying embolic signals with this technique [27]. These advantages and also increased RLSh detection rate likely contribute to the higher correlation with 'pathogenicity' as determined by the RoPE score.

To our knowledge, our analysis represents the largest cohort ever reported of patients with CS investigated by both c-TCD and TEE. As previously documented [20, 28], we demonstrated moderate agreement between c-TCD and TEE for identifying and grading RLSh. While prior work has suggested that TCD may be a sensitive tool compared to TEE $[17,29-33]$, these prior studies were not performed on large numbers of patients with CS and there is no prior work showing that TCD might be better at distinguishing pathogenically important from less important PFOs. In this analysis, the subset of patients investigated by both techniques was not representative of the entire CODICIA database. The restricted TEE ordering pathway discussed in our methods led to a cohort that disproportionally excluded patients with a low RoPE score, and therefore, did not afford the opportunity to directly compare these techniques with respect to correlation to the RoPE score. At this time, TEE continues to be the gold standard in the cardiac study of CS, since this technique allows direct visualization of the interatrial septum and allows for the detection of other potential sources of stroke including intracardiac thrombus and aortic atheroma - an advantage over c-TCD.

There were certain limitations of our study that should be noted. RLSh identified during c-TCD study may be due to extracardiac shunting (and not related to a PFO). This may contribute to the increased sensitivity in detecting RLSh of c-TCD compared to TEE found in this and other studies $[19,33,34]$. While the RoPE Score was derived from data from multiple databases, the TEE and c-TCD data for this analysis were derived only from the CODICIA database; therefore, this is a multicentered, but not a multinational study; the applicability to other centers around the world is not known. We did not evaluate the relationship between c-TCD shunt severity and stroke recurrence though a recent report from the RoPE study group indicates that there is significant mechanistic heterogeneity for stroke recurrence for patients with CS and PFO [35]. An additional limitation of selection bias might stem from the original CODICIA study protocol. As originally described, c-TCD was the reference evaluation of RLSh. TEE was obtained only if there was massive RLSh or if requested by the treating clinician. As a result, patients with small RLSh were less often studied with TEE. This observation has the potential to affect the correlation between these techniques. Finally, as is common in routine clinical practice, microbubble injection in the CODICIA study was performed from the anticubital vein, a site that correlates less well with anatomic size [36]. This may attenuate the correlation of c-TCD shunt grade with the RoPE score under more ideal conditions.

Nevertheless, we validated the RoPE score in this population and identified a correlation between RLSh severity assessed by the c-TCD and RoPE score, suggesting that this technique is an important tool for evaluating patients with CS. There is agreement between RLSh detection and grading with TEE and c-TCD though there are important examples of substantial right-to-left shunting seen only with c-TCD. More work is needed to identify and validate reproducible techniques for assessing the risk associated with observed RLSh for patients with CS.

\section{Disclosure Statement}

This study was partially funded by grants and supported by NIH (UL1 TR001064, 1TL1 TR001062, R01 NS062153, R21 NS079826), all from the National Institutes of Health. David E. Thaler and David M. Kent have consulted for WL Gore Associates. David E. Thaler is a consultant to AGA Medical Corporation. There are no other conflicts of interest to disclose.

\section{References}

1 Kutty S, Sengupta PP, Khandheria BK: Patent foramen ovale: the known and the to be known. J Am Coll Cardiol 2012;59:1665-1671.

2 Sacco RL, Ellenberg JH, Mohr JP, Tatemichi TK, Hier DB, Price TR, et al: Infarcts of undetermined cause: the NINCDS stroke data bank. Ann Neurol 1989;25:382-390.

3 Kent DM, Thaler DE: Is patent foramen ovale a modifiable risk factor for stroke recurrence? Stroke 2010;41(10 suppl):S26-S30.
4 Meier B, Kalesan B, Mattle HP, Khattab AA, Hildick-Smith D, Dudek D, et al: Percutaneous closure of patent foramen ovale in cryptogenic embolism. N Engl J Med 2013;368: 1083-1091.

5 Carroll JD, Saver JL, Thaler DE, Smalling RW, Berry S, MacDonald LA, et al: Closure of patent foramen ovale versus medical therapy after cryptogenic stroke. N Engl J Med 2013; 368:1092-1100.
6 Messé SR, Kent DM: Still no closure on the question of PFO closure. N Engl J Med 2013; 368:1152-1153.

7 Furlan AJ, Reisman M, Massaro J, Mauri L, Adams H, Albers GW, et al: Closure or medical therapy for cryptogenic stroke with patent foramen ovale. N Engl J Med 2012;366:991-999.

8 Alsheikh-Ali AA, Thaler DE, Kent DM: Patent foramen ovale in cryptogenic stroke: incidental or pathogenic? Stroke 2009;40:2349-2355.
Significance of Right-to-Left Shunting by TCD: Analysis of the CODICIA Database
Cerebrovasc Dis 2015;40:52-58 DOI: $10.1159 / 000430998$ 
9 Kent DM, Ruthazer R, Weimar C, Mas JL, Serena J, Homma S, et al: An index to identify stroke-related vs incidental patent foramen ovale in cryptogenic stroke. Neurology 2013; 81:619-625.

10 Agmon Y, Khandheria BK, Meissner I, Gentile F, Whisnant JP, Sicks JD, et al: Frequency of atrial septal aneurysms in patients with cerebral ischemic events. Circulation 1999;99: 1942-1944.

11 Lee JY, Song JK, Song JM, Kang DH, Yun SC, Kang DW, et al: Association between anatomic features of atrial septal abnormalities obtained by omni-plane transesophageal echocardiography and stroke recurrence in cryptogenic stroke patients with patent foramen ovale. Am J Cardiol 2010;106:129-134.

12 Rigatelli G, Dell'Avvocata F, Cardaioli P, Giordan M, Braggion G, Aggio S, et al: Permanent right-to-left shunt is the key factor in managing patent foramen ovale. J Am Coll Cardiol 2011;58:2257-2261.

13 Cabanes L, Coste J, Derumeaux G, Jeanrenaud $\mathrm{X}$, Lamy C, Zuber M, et al: Interobserver and intraobserver variability in detection of patent foramen ovale and atrial septal aneurysm with transesophageal echocardiography. J Am Soc Echocardiogr 2002;15:441-446.

14 Di Tullio MR: Patent foramen ovale: echocardiographic detection and clinical relevance in stroke. J Am Soc Echocardiogr 2010;23:144155; quiz 220.

15 Wessler BS, Thaler DE, Ruthazer R, Weimar C, Di Tullio MR, Elkind MS et al: Transesophageal echocardiography in cryptogenic stroke and patent foramen ovale: analysis of putative high-risk features from the risk of paradoxical embolism database. Circ Cardiovasc Imaging 2014;7:125-131.

16 Ringelstein EB, Droste DW, Babikian VL, Evans DH, Grosset DG, Kaps M, et al: Consensus on microembolus detection by TCD. International consensus group on microembolus detection. Stroke 1998;29:725-729.

17 Droste DW, Kriete JU, Stypmann J, Castrucci M, Wichter T, Tietje R, et al: Contrast transcranial Doppler ultrasound in the detection of right-to-left shunts: comparison of different procedures and different contrast agents. Stroke 1999;30:1827-1832.

18 Zito C, Dattilo G, Oreto G, Di Bella G, Lamari A, Iudicello R, et al: Patent foramen ovale: comparison among diagnostic strategies in cryptogenic stroke and migraine. Echocardiography 2009;26:495-503.
19 Mojadidi MK, Roberts SC, Winoker JS, Romero J, Goodman-Meza D, Gevorgyan R, et al: Accuracy of transcranial Doppler for the diagnosis of intracardiac right-to-left shunt: a bivariate meta-analysis of prospective studies. JACC Cardiovasc Imaging 2014;7:236-250.

20 Komar M, Olszowska M, Przewłocki T, Podolec J, Stępniewski J, Sobień B, et al: Transcranial Doppler ultrasonography should it be the first choice for persistent foramen ovale screening? Cardiovasc Ultrasound 2014;12:16.

21 Serena J, Marti-Fàbregas J, Santamarina E, Rodríguez JJ, Perez-Ayuso MJ, Masjuan J, et al: Recurrent stroke and massive right-to-left shunt: results from the prospective Spanish multicenter (CODICIA) study. Stroke 2008; 39:3131-3136.

22 Serena J, Segura T, Perez-Ayuso MJ, Bassaganyas J, Molins A, Dávalos A: The need to quantify right-to-left shunt in acute ischemic stroke: a case-control study. Stroke 1998;29: 1322-1328.

23 Jauss M, Zanette E: Detection of right-to-left shunt with ultrasound contrast agent and transcranial Doppler sonography. Cerebrovasc Dis 2000;10:490-496.

24 Hahn RT, Abraham T, Adams MS, Bruce CJ, Glas KE, Lang RM, et al: Guidelines for performing a comprehensive transesophageal echocardiographic examination: recommendations from the American society of echocardiography and the society of cardiovascular anesthesiologists. J Am Soc Echocardiogr 2013;26:921-964.

25 Kent DM, Thaler DE: The risk of paradoxical embolism (RoPE) study: developing risk models for application to ongoing randomized trials of percutaneous patent foramen ovale closure for cryptogenic stroke. Trials 2011;12:185.

26 Pfleger S, et al: Haemodynamic quantification of different provocation manoeuvres by simultaneous measurement of right and left atrial pressure: implications for the echocardiographic detection of persistent foramen ovale. Eur J Echocardiogr 2001;2:88-93.

27 Markus H, Bland JM, Rose G, Sitzer M, Siebler M: How good is intercenter agreement in the identification of embolic signals in carotid artery disease? Stroke 1996;27:12491252.

28 González-Alujas T, Evangelista A, Santamarina $\mathrm{E}$, Rubiera $\mathrm{M}$, Gómez-Bosch Z, Rodríguez-Palomares JF, et al: Diagnosis and quantification of patent foramen ovale. Which is the reference technique? Simultaneous study with transcranial Doppler, transthoracic and transesophageal echocardiography. Rev Esp Cardiol 2011;64:133-139.

29 Caputi L, Carriero MR, Falcone C, Parati E, Piotti P, Materazzo C, et al: Transcranial Doppler and transesophageal echocardiography: comparison of both techniques and prospective clinical relevance of transcranial Doppler in patent foramen ovale detection. J Stroke Cerebrovasc Dis 2009;18:343-348.

30 Jauss M, Kaps M, Keberle M, Haberbosch W, Dorndorf W: A comparison of transesophageal echocardiography and transcranial Doppler sonography with contrast medium for detection of patent foramen ovale. Stroke 1994;25:1265-1267.

31 Job FP, Ringelstein EB, Grafen Y, Flachskampf FA, Doherty C, Stockmanns A, et al: Comparison of transcranial contrast Doppler sonography and transesophageal contrast echocardiography for the detection of patent foramen ovale in young stroke patients. Am J Cardiol 1994;74:381-384.

32 Droste DW, Lakemeier H, Ritter M, Dittrich $\mathrm{R}$, Stypmann J, Wichter T, et al: The identification of right-to-left shunts using contrast transcranial Doppler ultrasound: performance and interpretation modalities, and absence of a significant side difference of cardiac micro-emboli. Neurol Res 2004;26:325-330.

33 Souteyrand G, Motreff P, Lusson JR, Rodriguez R, Geoffroy E, Dauphin C, et al.: Comparison of transthoracic echocardiography using second harmonic imaging, transcranial Doppler and transesophageal echocardiography for the detection of patent foramen ovale in stroke patients. Eur J Echocardiogr 2006;7: 147-154.

34 Goutman SA, Katzan IL, Gupta R: Transcranial Doppler with bubble study as a method to detect extracardiac right-to-left shunts in patients with ischemic stroke. J Neuroimaging 2013;23:523-525.

35 Thaler DE, Ruthazer R, Weimar C, Mas JL, Serena J, Di Angelantonio E, et al: Recurrent stroke predictors differ in medically treated patients with pathogenic vs. other PFOs. Neurology 2014;83:221-226.

36 Schuchlenz HW, Weihs W, Beitzke A, Stein JI, Gamillscheg A, Rehak P: Transesophageal echocardiography for quantifying size of patent foramen ovale in patients with cryptogenic cerebrovascular events. Stroke 2002;33: 293-296. 